[23] E. Viscito and J. P. Allebach, "Design of perfect reconstruction multidimensional filter banks using cascaded Smith form matrices," in Proc. 1988 IEEE Int. Symp. Circuits Syst., vol. 1, (Helsinki, Finland), pp. 831-834, June 1988.

[24] D. C. Farden and L. L. Scharf, "Statistical design of nonrecursive digital filters," IEEE Trans. Acoust., Speech, Signal Process., pp. 188-196, June 1974.

[25] T. Q. Nguyen and P. P. Vaidyanathan, "Maximally decimated perfectreconstruction FIR filter banks with pairwise mirror-image analysis (and synthesis) frequency response," IEEE Trans. Acoust., Speech, Signal Process., pp. 693-706, May 1988.

[26] V. C. Liu and P. P. Vaidyanathan, "On the factorization of a subclass of 2-D digital FIR lossless matrices for 2-D QMF bank applications," IEEE Trans. Circuits Syst., vol. 37, pp. 852-854, June 1990.

[27] S. Y. Kung, B. C. Levy, M. Morf, and T. Kailath, "New results in 2 D systems theory, Part II: 2-D state-space models-realization and the notions of controllability, observability and minimality," Proc. IEEE, vol. 65, pp. 945-961, June 1977.

[28] C. S. Koo and C. T. Chen, "Faddeeva's algorithm for spatial dynamical equations," Proc. IEEE, vol. 65, pp. 975-976, June 1977.

[29] E. Simoncelli and E. H. Adelson, "Subband transforms," in Subband Image Coding, J. W. Woods, Ed. Boston: Kluwer Academic, 1991, pp. 143-192.

\section{Noncausal Image Modeling Using Descriptor Approach}

\author{
Mohammed A. Hasan and Mahmood R. Azimi-Sadjadi
}

Abstract-The problems of noncausal image modeling and subsequen image estimation are considered in this brief. The noncausal vector autoregressive (AR) model for the image process is arranged into a descriptor system. This system is then decomposed into backward and forward stable subsystems. The resulting subsystems are utilized to derive a Kalman filter by solving some types of discrete time algebraic Liapunov equations. A numerical example for noncausal image modeling is also presented.

\section{INTRODUCTION}

The problem of image modeling involves fitting an appropriate autoregressive (AR) or autoregressive moving average (ARMA) model to the image data. Unlike most 1-D signals that are typically time dependent, images are defined in spatial coordinates and thus causality is not a rigid requirement for the physical realizability of the image model. In fact, the region of support (ROS) of an image model can be causal, semicausal, or noncausal leading to initial value, initial-boundary value, or boundary-value problems [1], respectively.

In almost all the previous treatments [2]-[4], causal ROS was mainly used. However, by using causal models, typically close to half of the adjoining pixels in the ROS were ignored in the process of modeling. On the other hand, in noncausal modeling, correlational information is extracted from as many pixels surrounding a given pixel as possible and thus the correlations of a particular pixel with al spatially close pixels are reflected in the model. Therefore, one would expect that noncausal models provide better representation than the causal ones. This naturally leads to a more accurate filtering operation.

Until very recently image modeling and restoration using noncausal image and blur modeling was not fully investigated in the literature. However, recently there has been some interest in noncausal AR and

Manuscript received June 21, 1993; revised May 27, 1994 and November 8, 1994. This paper was recommended by Associate Editor W.-S. Lu.

The authors are with the Department of Electrical Engineering, Colorado State University, Fort Collins, CO 80523 USA

IEEE Log Number 9413188

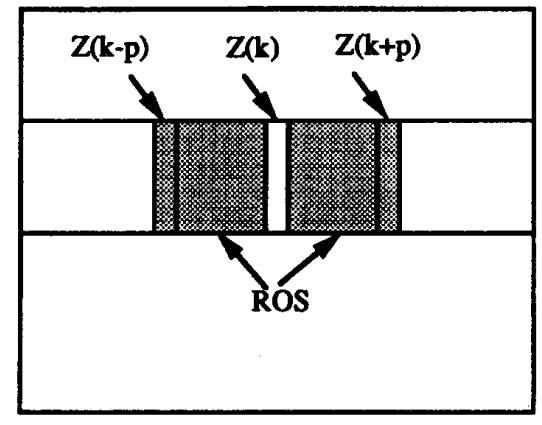

Fig. 1. ROS of noncausal model.

ARMA models mainly because noncausal models are more natural choices for many applications. In [5], [6] it is indicated that noncausal processes can be described by descriptor systems. Recursive processing of 1-D and 2-D noncausal systems is reported in [7]. In [8], 2-D spectrum estimation using noncausal models is presented. In [9], a technique for modeling and recursive state estimation for 2-D noncausal filters was proposed. Identification and restoration of images degraded by symmetric noncausal blur is treated in [10].

Perhaps the most natural way of dealing with noncausal discrete time models is through the descriptor form [5], [6]. The main goal of this brief is to develop a scheme using descriptor approach to represent an image modeled by a vector AR process with a noncausal ROS. The Kronecker canonical form decomposition is then applied to this descriptor system yielding two subsystems which perform equivalent to the original system. These subsystems may then be used to develop a Kalman filter for image restoration applications.

\section{MODELING OF IMAGE AND DEgRADATION PROCESSES IN DESCRIPTOR FORM}

Consider an $N \times N$ image which is scanned horizontally in strips of width $W$. It is assumed that the image is column widesense stationary [4] within each strip. Let $Z(k)$ denote the $k$-th, $k \in[0, N-1]$, image vector of size $W$ in the $i$-th strip, i.e.,

$$
Z(k)=\left[z_{(i-1) W, k}, z_{(i-1) W+1, k} \cdots z_{i W-1, k}\right]^{T}
$$

where $z_{m, n}$ represents the intensity value of the original image at location $(m, n)$ and the superscript $T$ denotes matrix transposition. Assume that the image process is modeled by a minimum variance representation (MVR) AR noncausal process of order $p$,

$$
\begin{aligned}
Z(k)= & \Phi_{p}^{T} Z(k-p)+\cdots+\Phi_{1}^{T} Z(k-1) \\
& +\Phi_{-1}^{T} Z(k+1)+\cdots+\Phi_{-p}^{T} Z(k+p)+U(k)
\end{aligned}
$$

where $\Phi_{-p}, \Phi_{-p+1}, \cdots \Phi_{p}$ are constant $W \times W$ autoregressive parameter matrices. The ROS of this model is shown in Fig. 1.

The vector $U(k)$ is a $W \times 1$ zero mean noise vector driving the AR process. The necessary and sufficient conditions for the covariance matrix $Q_{U}:=E\left[U(k) U^{T}(k)\right]$ to be minimum is that the orthogonal property, $E\left[U(k-l) Z^{T}(k)\right]=Q_{U} \delta(l)$, holds for $l \in[-p, p]$. This orthogonality principle leads to the following normal equations

$\rho_{r}=\rho_{r-p} \Phi_{p}+\cdots+\rho_{r-1} \Phi_{1}+\rho_{r+1} \Phi_{-1}+\cdots+\rho_{r+p} \Phi_{-p}+Q_{U} \delta(r)$

for $r=-p, \cdots,-1,0,1, \cdots, p$, where $\rho_{r}:=E\left[Z(k-r) Z^{T}(k)\right]$ which can be solved to yield the AR parameter matrices. Note that $\rho_{-r}=\rho_{r}^{T}$. 
It should be noted that in contrary to the causal AR models which are driven by white noise process, noncausal MVR models are driven by colored noise process [1]. This can be shown by multiplying both sides of (1) by $U(k-l)$ and taking expectation, which yields

$$
E\left[U(k-l) U^{T}(k)\right]=Q_{U}\left\{\delta(l)-\sum_{\substack{i=-p \\ i \neq 0}}^{p} \Phi_{i} \delta(l-i)\right\} .
$$

This implies that $U(k)$ is a colored process since $E[U(k-$ $\left.l) U^{T}(k)\right] \neq 0$ for $l \in[-p, p], l \neq 0$.

\section{1) Descriptor State-Space Model}

The noncausal vector AR model in (1) can be transformed into a descriptor system using the following local state vector

$$
\bar{X}(k)=\left[x_{p}(k)^{T}, \cdots, x_{1}^{T}(k), x_{0}^{T}(k), x_{-1}^{T}(k), \cdots, x_{-p}^{T}(k)\right]^{T}
$$

where $x_{i}(k)=Z(k-i)$ for $i=-p, \cdots, p$. This state assignment yields the following "descriptor" or "singular" system [5], [6]

$$
\begin{aligned}
\bar{E} \bar{X}(k+1) & =\bar{A} \bar{X}(k)+\bar{B} U(k) \\
x_{0}(k)=Z(k) & =\bar{C} \bar{X}(k)
\end{aligned}
$$

where (see (4b) below). $\bar{C}=\left[\begin{array}{lllllll}0 & \cdots & 0 & I_{W} & 0 & \cdots & 0\end{array}\right]$ and $\bar{B}=\left[\begin{array}{lllll}0 & 0 & \cdots & 0 & I_{W}\end{array}\right]^{T}$. Note that $\bar{E}$ and $\bar{A}$ are $(2 p+1) W \times$ $(2 p+1) W$ matrices with $\bar{E}$ being singular of rank $2 p \times W$ and $\bar{B}^{T}$ and $\bar{C}$ are $W \times(2 p+1) W$ matrices. It can be shown [10] that the vector AR model (1) and the descriptor system (4a) have the same transfer function matrix. A by-product of this result is that the pencil $z \bar{E}-\bar{A}$ is regular [11], since from (1) the determinant $|z \bar{E}-\bar{A}| \neq 0$. It can be shown that $p W$ zeros of this polynomial $p(z)=|z \bar{E}-\bar{A}|=0$ are inside the unit circle while the other $p W$ zeros are outside the unit circle.

Although $U(k)$ in (4a) is not white, it can be modeled by a finite dimensional linear system [12] driven by a white process as

$$
\begin{aligned}
V(k+1) & =A_{a} V(k)+B_{a} w(k) \\
U(k) & =C_{a} V(k)
\end{aligned}
$$

where $w(k)$ is a zero mean white vector process and $A_{a}, B_{a}$ and $C_{a}$ are matrices of appropriate dimensions. Augmenting this system with system $(4 a)$ yields

$$
\begin{aligned}
E X(k+1) & =A X(k)+B w(k) \\
Z(k) & =C X(k)
\end{aligned}
$$

where $X(k):=\left[\begin{array}{l}\bar{X}(k) \\ V(k)\end{array}\right], A:=\left[\begin{array}{cc}\bar{A} & \bar{B} C_{a} \\ 0 & A_{a}\end{array}\right], E:=\left[\begin{array}{cc}\bar{E} & 0 \\ 0 & I\end{array}\right]$, $B:=\left[\begin{array}{c}0 \\ B_{a}\end{array}\right]$ and $C:=\left[\begin{array}{ll}\bar{C} & 0\end{array}\right]$.

The degradation due to a full-plane blur [9] and additive white Gaussian noise can be modeled as $y(k)=\bar{H} \bar{X}(k)+v(k)$, where $\{v(k)\}$ is zero mean white process and $\bar{H}$ consists of the elements of the point spread function (PSF) of the full-plane blur. Combining the image and degradation processes gives the following descriptor system

$$
\begin{aligned}
E X(k+1) & =A X(k)+B w(k) \\
y(k) & =H X(k)+v(k)
\end{aligned}
$$

where $H=\left[\begin{array}{ll}\bar{H} & 0\end{array}\right]$. In the state estimation for (7) the boundary conditions $X(0)$ and $X(N-1)$ are needed and can be taken to be $\left[\begin{array}{lll}y^{T}(0) & \cdots & y(2 p)^{T}, 0\end{array}\right]^{T}$ and $\left[\begin{array}{lll}y^{T}(N-1-2 p) & \cdots & y(N-1)^{T}, 0\end{array}\right]^{T}$ where $y(0), \cdots, y(2 p)$ and $y(N-1-2 p), \cdots, y(N-1)$ are the first and last $(2 p+1)$ columns of the strip under consideration.

To develop state estimator for system (7) it is useful to transform the system into two stable subsystems using the Kronecker canonical form as described next.

\section{2) Stable Forward-Backward Decomposition}

Descriptor systems in general are not stable. In digital applications of descriptor systems it is necessary to decompose them into stable subsystems. This can be established as in the following theorem.

Theorem 1 [10]: Let $E, A \in \mathbb{R}^{n \times n}$, then there are two nonsingular real matrices $P$ and $Q$ such that $P E Q=\left[\begin{array}{cc}I_{n_{1}} & 0 \\ 0 & A_{b}\end{array}\right]$ and $P A Q=\left[\begin{array}{cc}A_{f} & 0 \\ 0 & I_{n_{2}}\end{array}\right]$ where $n_{1}, n_{2} \geq 0, n_{1}+n_{2}=n$, and $A_{f}$ and $A_{b}$ are stable matrices.

Note that if $|z E-A|$ has no zeros on the unit circle, then the matrices $A_{f}$ and $A_{b}$ in Theorem 1 are strictly stable. Applying this decomposition to the system (7) yields

$$
\begin{aligned}
X_{f}(k+1) & =A_{f} X_{f}(k)+B_{f} w(k) \\
X_{b}(k) & =A_{b} X_{b}(k+1)-B_{b} w(k) \\
y(k) & =H_{f} X_{f}(k)+H_{b} X_{b}(k)+v(k)
\end{aligned}
$$

where $\left[\begin{array}{l}X_{f}(k) \\ X_{b}(k)\end{array}\right]:=Q^{-1} X(k),\left[\begin{array}{l}B_{f} \\ B_{b}\end{array}\right]:=P B$ and $\left[\begin{array}{ll}H_{f} & H_{b}\end{array}\right]:=$ $H Q$.

This system is equivalent to the following forward and backward subsystems, respectively, propagating in opposite directions:

$$
\begin{gathered}
X_{f}(k+1)=A_{f} X_{f}(k)+B_{f} w(k) \\
y(k)=H_{f} X_{f}(k)+v_{f}(k)
\end{gathered}
$$

and

$$
\begin{aligned}
X_{b}(k) & =A_{b} X_{b}(k+1)-B_{b} w(k) \\
y(k) & =H_{b} X_{b}(k)+v_{b}(k) .
\end{aligned}
$$

Note that the measurement noise in the forward and backward subsystems (9a) and (9b) are $v_{f}(k)=H_{b} X_{b}(k)+v(k)$ and $v_{b}(k)=$ $H_{f} X_{f}(k)+v(k)$, respectively, which are colored.

The following example illustrates how a noncausal vector model can be decomposed into stable forward-backward subsystems using the method developed in this paper.

Example: Let us consider a zero-mean stationary image with autocorrelation function $r_{z}(j, l)=E\left[z_{i-j, k-1} z_{i, k}\right]=\rho^{|j|+|l|}$, where $|\rho|<1$. This image is to be represented by a first-order noncausal vector $\mathrm{AR}$ model with $W=2$. The parameter matrices can be determined by solving the following normal equations for $r=$ $-1,0,1$

$$
\rho_{r}=\rho_{r-1} \Phi_{1}+\rho_{r+1} \Phi_{1}+Q_{U} \delta(r)
$$

$$
\bar{E}=\left(\begin{array}{ccccc}
I_{W} & 0 & \cdots & 0 & 0 \\
0 & I_{W} & \cdots & 0 & 0 \\
\ldots \ldots & \ldots & \ldots & \ldots \ldots & \ldots \\
0 & 0 & \cdots & I_{W} & 0 \\
0 & 0 & \cdots & 0 & 0
\end{array}\right), \quad \bar{A}=\left(\begin{array}{cccccc}
0 & I_{W} & 0 & \cdots & 0 & 0 \\
0 & 0 & I_{W} & \cdots & 0 & 0 \\
\ldots \ldots \ldots \ldots & \ldots \ldots & \ldots & \ldots & \ldots \\
0 & 0 & \cdots & 0 & \ldots & I_{W} \\
\Phi_{p}^{T} & \Phi_{\mathrm{p}-1}^{T} & \cdots & -I_{W} & \cdots & \Phi_{-p}^{T}
\end{array}\right)
$$


where $\rho_{r}:=E\left[Z(k-r) Z^{T}(k)\right]$ and $Z(k)=\left[z_{2 i-2, k}, z_{2 i-1, k}\right]^{T}$, i.e.,

$$
\begin{aligned}
\rho_{1} & =\rho_{0} \Phi_{1}+\rho_{2} \Phi_{-1} \\
\rho_{0} & =\rho_{-1} \Phi_{1}+\rho_{1} \Phi_{-1}+Q_{U} \\
\rho_{-1} & =\rho_{-2} \Phi_{1}+\rho_{0} \Phi_{-1} .
\end{aligned}
$$

Note that the correlation matrices $\rho_{\mathrm{r}}$ are given by $\rho_{r}=$ $\left[\begin{array}{cc}\rho^{|r|} & \rho^{|r|+1} \\ \rho^{|r|+1} & \rho^{|r|}\end{array}\right]=\rho^{|r|}\left[\begin{array}{ll}1 & \rho \\ \rho & 1\end{array}\right]$ and $\rho_{-r}=\rho_{r}^{T}$.

The matrices $\phi_{1}$ and $\phi_{-1}$ are solutions of the following system

$$
\left[\begin{array}{cc}
\rho_{0} & \rho_{2} \\
\rho_{2}^{T} & \rho_{0}
\end{array}\right]\left[\begin{array}{c}
\phi_{1} \\
\phi_{-1}
\end{array}\right]=\left[\begin{array}{c}
\rho_{1} \\
\rho_{1}^{T}
\end{array}\right]
$$

from which it follows that

$$
\left[\begin{array}{c}
\phi_{1} \\
\phi_{-1}
\end{array}\right]=\left[\begin{array}{cccc}
1 & \rho & \rho^{2} & \rho^{3} \\
\rho & 1 & \rho^{3} & \rho^{2} \\
\rho^{2} & \rho^{3} & 1 & \rho \\
\rho^{3} & \rho^{2} & \rho & 1
\end{array}\right]^{-1}\left[\begin{array}{cc}
\rho & \rho^{2} \\
\rho^{2} & \rho \\
\rho & \rho^{2} \\
\rho^{2} & \rho
\end{array}\right]=\frac{\rho}{1+\rho^{2}}\left[\begin{array}{ll}
1 & 0 \\
0 & 1 \\
1 & 0 \\
0 & 1
\end{array}\right],
$$

hence $\phi_{1}=\phi_{-1}=\frac{\rho}{1+\rho^{2}} I$. Solving the third equation of $(10)$ for $Q_{U}$ yields $Q_{U}=\frac{1-\rho^{2}}{1+\rho^{2}}\left[\begin{array}{ll}1 & \rho \\ \rho & 1\end{array}\right]$. As a result, the first order noncausal vector AR model with $W=2$ has the following form

$$
Z(k)=\left[\begin{array}{cc}
\frac{\rho}{1+\rho^{2}} & 0 \\
0 & \frac{\rho}{1+\rho^{2}}
\end{array}\right] Z(k-1)+\left[\begin{array}{cc}
\frac{\rho}{1+\rho^{2}} & 0 \\
0 & \frac{\rho}{1+\rho^{2}}
\end{array}\right] Z(k+1)+U(k)
$$

where $U(k)=\left[u_{2 i-2, k}, u_{2 i-1, k}\right]^{T}$. Note that this vector model is equivalent to two 1-D models driven by colored noise. Arranging this model into a descriptor system gives

$$
\begin{aligned}
\bar{E} \bar{X}(k+1) & =\bar{A} \bar{X}(k)+\bar{B} U(k) \\
Z(k) & =\bar{C} \bar{X}(k)
\end{aligned}
$$

where $\bar{X}(k)=\left[Z^{T}(k-1), Z^{T}(k), Z^{T}(k+1)\right]^{T}$, (see first matrix at the bottom of the page), $\bar{B}=\left[\begin{array}{cccccc}0 & 0 & 0 & 0 & 1 & 0 \\ 0 & 0 & 0 & 0 & 0 & 1\end{array}\right]^{T}$ and $\bar{C}=\left[\begin{array}{llllll}0 & 0 & 1 & 0 & 0 & 0 \\ 0 & 0 & 0 & 1 & 0 & 0\end{array}\right]$.

The generalized eigenvalues which are solutions of $|\lambda \bar{E}-\bar{A}|=0$ are found to be $\lambda=\rho, \rho, \frac{1}{\rho}, \frac{1}{\rho}, 0,0$, i.e., four eigenvalues are inside the unit circle while the other two are outside. Using the procedure in [11] and the results of Theorem 1 we can define transformation matrices $P$ and $Q$ such that the descriptor system for the noncausal image model is decomposed into stable forwardbackward subsystems. These matrices are found to be (See second matrix at the bottom of the page.)

Now, it can easily be checked that $P \bar{E} Q=\left[\begin{array}{cc}I_{2} & 0 \\ 0 & \bar{A}_{b}\end{array}\right]$ and $P \bar{A} Q=\left[\begin{array}{cc}\bar{A}_{f} & 0 \\ 0 & I_{4}\end{array}\right]$, where $\bar{A}_{f}=\left[\begin{array}{cc}\rho & 0 \\ 0 & \rho\end{array}\right], \bar{A}_{b}=\left[\begin{array}{cccc}\rho & 0 & 0 & 0 \\ 0 & \rho & 0 & 0 \\ 0 & 0 & 0 & 0 \\ 0 & 0 & 0 & 0\end{array}\right]$, $I_{2}$ and $I_{4}$ are $2 \times 2$ and $4 \times 4$ identity matrices, respectively. Therefore, we obtain the following decoupled stable subsystems

$$
\begin{aligned}
\bar{X}_{f}(k+1) & =\left[\begin{array}{ll}
\rho & 0 \\
0 & \rho
\end{array}\right] \bar{X}_{f}(k)+\left[\begin{array}{cc}
\frac{1+\rho^{2}}{\rho} & 0 \\
0 & \frac{1+\rho^{2}}{\rho}
\end{array}\right] U(k) \\
\bar{X}_{b}(k) & =\left[\begin{array}{llll}
\rho & 0 & 0 & 0 \\
0 & \rho & 0 & 0 \\
0 & 0 & 0 & 0 \\
0 & 0 & 0 & 0
\end{array}\right] \bar{X}_{b}(k+1)
\end{aligned}
$$$$
+\left[\begin{array}{cc}
\frac{1+\rho^{2}}{1-\rho^{2}} & 0 \\
0 & \frac{1+\rho^{2}}{1-\rho^{2}} \\
\frac{-\rho\left(1+\rho^{2}\right)}{1-\rho^{2}} & 0 \\
0 & \frac{-\rho\left(1+\rho^{2}\right)}{1-\rho^{2}}
\end{array}\right] U(k)
$$

$$
Z(k)=\left[\begin{array}{cc}
\frac{1}{\rho} & 0 \\
0 & \frac{1}{\rho}
\end{array}\right] \bar{X}_{f}(k)+\left[\begin{array}{cccc}
\rho & 0 & 0 & 0 \\
0 & \rho & 0 & 0
\end{array}\right] \bar{X}_{b}(k)
$$

where $\left[\begin{array}{l}\bar{X}_{f} \\ \bar{X}_{b}\end{array}\right]=Q^{-1} \bar{X}(k)$ and

$$
Q^{-1}=\left[\begin{array}{cccccc}
\frac{1}{1-\rho^{2}} & 0 & \frac{-\rho}{1-\rho^{2}} & 0 & 0 & 0 \\
0 & \frac{1}{1-\rho^{2}} & 0 & \frac{-\rho}{\rho\left(1-\rho^{2}\right)} & 0 & 0 \\
\frac{-\rho^{2}}{1-\rho^{2}} & 0 & \frac{\rho}{1-\rho^{2}} & 0 & 0 & 0 \\
0 & \frac{-\rho^{2}}{1-\rho^{2}} & 0 & \frac{\rho}{1-\rho^{2}} & 0 & 0 \\
1 & 0 & -\frac{1+\rho^{2}}{\rho} & 0 & 1 & 0 \\
0 & 1 & 0 & -\frac{1+\rho^{2}}{\rho} & 0 & 1
\end{array}\right] .
$$

As can be observed, all eigenvalues of the forward and backward subsystems are at $\rho,(|\rho|<1)$, with the exception of two eigenvalues of the backward subsystem which are at the origin of the unit circle, i.e., deadbeat. In addition, since no approximation is used in computing $P$ and $Q$ matrices, the subsystems preserve all the correlational information of the original model.

$$
\bar{E}=\left[\begin{array}{llllll}
1 & 0 & 0 & 0 & 0 & 0 \\
0 & 1 & 0 & 0 & 0 & 0 \\
0 & 0 & 1 & 0 & 0 & 0 \\
0 & 0 & 0 & 1 & 0 & 0 \\
0 & 0 & 0 & 0 & 0 & 0 \\
0 & 0 & 0 & 0 & 0 & 0
\end{array}\right], \quad \bar{A}=\left[\begin{array}{cccccc}
0 & 0 & 1 & 0 & 0 & 0 \\
0 & 0 & 0 & 1 & 0 & 0 \\
0 & 0 & 0 & 0 & 1 & 0 \\
0 & 0 & 0 & 0 & 0 & 1 \\
\frac{\rho}{1+\rho^{2}} & 0 & -1 & 0 & \frac{\rho}{1+\rho^{2}} & 0 \\
0 & \frac{\rho}{1+\rho^{2}} & 0 & -1 & 0 & \frac{\rho}{1+\rho^{2}}
\end{array}\right]
$$

$$
P=\left[\begin{array}{cccccc}
\frac{1}{1-\rho^{2}} & 0 & \frac{-\rho}{1-\rho^{2}} & 0 & \frac{1+\rho^{2}}{1-\rho^{2}} & 0 \\
0 & \frac{1}{1-\rho^{2}} & 0 & \frac{-\rho}{1-\rho^{2}} & 0 & \frac{1+\rho^{2}}{1-\rho^{2}} \\
\frac{-\rho^{3}}{1-\rho^{2}} & 0 & \frac{\rho^{2}}{1-\rho^{2}} & 0 & \frac{-\rho\left(1+\rho^{2}\right)}{1-\rho^{2}} & 0 \\
0 & \frac{-\rho^{3}}{1-\rho^{2}} & 0 & \frac{\rho^{2}}{1-\rho^{2}} & 0 & \frac{-\rho\left(1+\rho^{2}\right)}{1-\rho^{2}} \\
0 & 0 & 0 & 0 & \frac{1+\rho^{2}}{\rho} & 0 \\
0 & 0 & 0 & 0 & 0 & \frac{1+\rho^{2}}{\rho}
\end{array}\right], Q=\left[\begin{array}{cccccc}
1 & 0 & 1 & 0 & 0 & 0 \\
0 & 1 & 0 & 1 & 0 & 0 \\
\rho & 0 & \frac{1}{\rho} & 0 & 0 & 0 \\
0 & \rho & 0 & \frac{1}{\rho} & 0 & 0 \\
\rho^{2} & 0 & \frac{1}{\rho^{2}} & 0 & 1 & 0 \\
0 & \rho^{2} & 0 & \frac{1}{\rho^{2}} & 0 & 1
\end{array}\right]
$$


Even though the above procedure is only applied to the image model and the effects of colored noise and the degradation processes are not considered, the general approach of this brief can be utilized to decompose the complete system (7) into forward and backward subsystems and then derive the corresponding reduced order Kalman filters for image restoration applications.

\section{STATE Estimation FOR FORWARD-BACKWARD SUBSystemS}

In this section, we formulate the problem of state estimation for subsystems (9) into standard reduced order Kalman filters. To achieve this, state space models for the colored processes $v_{f}(k)$ and $v_{b}(k)$ are needed.

We start by obtaining the statistics of the processes $v_{f}(k)$ and $v_{b}(k)$ which can be computed using only the statistics of the zero mean white processes, $\{w(k)\}$ and $\{v(k)\}$, which are assumed to be known. Let $P_{f}:=E\left[X_{f}(k) X_{f}^{T}(k)\right]$ and $P_{b}:=E\left[X_{b}(k) X_{b}^{T}(k)\right]$ be the forward and backward state covariances, respectively. Then, using the state equation in (9a) and assuming stationarity, it can easily be shown that $P_{f}$ is the positive definite solution of the discrete Liapunov matrix equation $P_{f}=A_{f} P_{f} A_{f}^{T}+B_{f} Q_{w} B_{f}^{T}$ which is given by

$$
P_{f}=\sum_{k=0}^{\infty} A_{f}^{k} B_{f} Q_{w} B_{f}^{T} A_{f}^{T^{k}}
$$

where $Q_{w}:=E\left[w(k) w^{T}(k)\right]$. The limit of this summation exists as $A_{f}$ is stable [8]. As a result, we have $\rho_{i f}:=E\left[v_{f}(k) v_{f}^{T}(k+i)\right]=$ $Q_{v} \delta(i)+H_{b} A_{b}^{i} P_{b} H_{b}^{T}$ for $i=0,1,2 \cdots$.

Similarly using the backward state equation in (9b) yields $P_{b}=$ $A_{b} P_{b} A_{b}^{T}+B_{b} Q_{w} B_{b}^{T}$. Again the stability of $A_{b}$ ensures the existence of the following solution

$$
P_{b}=\sum_{k=0}^{\infty} A_{b}^{k} B_{b} Q_{w} B_{b}^{T} A_{b}^{T^{k}}
$$

It can readily be verified that $\rho_{i b}:=E\left[v_{b}(k) v_{b}^{T}(k+i)\right]=Q_{v} \delta(i)+$ $H_{f} P_{f} A_{f}^{T^{i}} H_{f}^{T}$ for $i=0,1, \cdots$.

Having determined the statistics of $v_{f}(k)$ and $v_{b}(k)$, we now model these processes by a $q$-th and $s$-th order AR models, respectively. Thus, for $v_{f}(k)$ we have

$$
v_{f}(k)=\phi_{1}^{T} v_{f}(k-1)+\cdots+\phi_{q}^{T} v_{f}(k-q)+\eta_{f}(k)
$$

where $\eta_{f}(k)$ is a zero mean white noise process with covariance matrix $Q_{\eta f}$. The parameters $\phi_{1}, \cdots, \phi_{q}$ are obtained by solving the following Yule-Walker system of equations

$$
\left[\begin{array}{cccc}
\rho_{0 f} & \rho_{1 f}^{T} & \ldots & \rho_{q f}^{T} \\
\rho_{1 f} & \rho_{0 f} & \ldots & \rho_{q-1 f}^{T} \\
\ldots \ldots & \ldots \ldots \ldots \ldots & \ldots \ldots \\
\rho_{q f} & \rho_{q-1 f} & \ldots & \rho_{0 f}
\end{array}\right]\left[\begin{array}{c}
-I \\
\phi_{1} \\
\vdots \\
\phi_{q-1} \\
\phi_{q}
\end{array}\right]=\left[\begin{array}{c}
-Q_{\eta_{f}} \\
\vdots \\
0 \\
0
\end{array}\right] .
$$

Once the model parameters are identified, the following state vector can be defined

$$
Z_{f}(k)^{T}=\left[v_{f}(k)^{T}, v_{f}(k-1)^{T}, \cdots, v_{f}(k-q+1)^{T}\right] .
$$

Then (13) can be written in the following state space form

$$
\begin{aligned}
Z_{f}(k+1) & =F_{f} Z_{f}(k)+G_{f} \eta_{f}(k) \\
v_{f}(k) & =C_{f} Z_{f}(k)
\end{aligned}
$$

where

$$
\begin{aligned}
& F_{f}=\left[\begin{array}{ccccc}
\phi_{1}^{T} & \phi_{2}^{T} & \cdots & \phi_{q-1}^{T} & \phi_{q}^{T} \\
I & 0 & \cdots & 0 & 0 \\
0 & I & \cdots & 0 & 0 \\
\ldots \ldots & \ldots \ldots \ldots \ldots \ldots \ldots \\
0 & 0 & \cdots & I & 0
\end{array}\right] \\
& G_{f}=\left[\begin{array}{lll}
I & 0 \cdots 0
\end{array}\right]^{T} \\
& C_{f}=\left[\begin{array}{lll}
I & 0 \cdots 0
\end{array}\right] \text {. }
\end{aligned}
$$

Augmenting systems (9a) and (15) yields

$$
\begin{aligned}
{\left[\begin{array}{c}
X_{f}(k+1) \\
Z_{f}(k+1)
\end{array}\right] } & =\left[\begin{array}{cc}
A_{f} & 0 \\
0 & F_{f}
\end{array}\right]\left[\begin{array}{c}
X_{f}(k) \\
Z_{f}(k)
\end{array}\right]+\left[\begin{array}{cc}
B_{f} & 0 \\
0 & G_{f}
\end{array}\right]\left[\begin{array}{c}
w(k) \\
\eta_{f}(k)
\end{array}\right] \\
y(k) & =\left[\begin{array}{ll}
H_{f} & C_{f}
\end{array}\right]\left[\begin{array}{l}
X_{f}(k) \\
Z_{f}(k)
\end{array}\right] .
\end{aligned}
$$

Similarly, we can model the process $v_{b}(k)$ by a finite dimensiona linear system of order $s$,

$$
v_{b}(k)=\psi_{1}^{T} v_{b}(k+1)+\cdots+\psi_{s}^{T} v_{b}(k+s)+\eta_{b}(k)
$$

where $\eta_{b}(k)$ is a zero mean white noise process with covariance matrix $Q_{\eta b}$. An analogous Yule-Walker system of equations can be arranged to yield the parameter matrices $\psi_{1}, \cdots, \psi_{s}$. Once the model parameters are identified, the following state vector can be defined

$$
Z_{b}(k)^{T}=\left[v_{b}(k)^{T}, v_{b}(k+1)^{T}, \cdots, v_{b}(k+s-1)^{T}\right] .
$$

Then (18) can be arranged into the following state space system

$$
\begin{aligned}
Z_{b}(k) & =F_{b} Z_{b}(k+1)+G_{b} \eta_{b}(k) \\
v_{b}(k) & =C_{b} Z_{b}(k)
\end{aligned}
$$

where

$$
\begin{aligned}
F_{b} & =\left[\begin{array}{ccccc}
\psi_{1}^{T} & \psi_{2}^{T} & \cdots & \psi_{s-1}^{T} & \psi_{s}^{T} \\
I & 0 & \cdots & 0 & 0 \\
0 & I & \cdots & 0 & 0 \\
\cdots \ldots & \ldots \ldots \ldots \ldots \ldots \ldots \\
0 & 0 & \cdots & I & 0
\end{array}\right] \\
G_{b} & =\left[\begin{array}{llll}
I & 0 & \cdots & 0
\end{array}\right]^{T} \\
C_{b} & =\left[\begin{array}{llll}
I & 0 & \cdots & 0
\end{array}\right] .
\end{aligned}
$$

Augmenting systems (9b) and (19) yields

$$
\begin{aligned}
{\left[\begin{array}{c}
X_{b}(k) \\
Z_{b}(k)
\end{array}\right] } & =\left[\begin{array}{cc}
A_{b} & 0 \\
0 & F_{b}
\end{array}\right]\left[\begin{array}{l}
X_{b}(k+1) \\
Z_{b}(k+1)
\end{array}\right]+\left[\begin{array}{cc}
-B_{b} & 0 \\
0 & G_{b}
\end{array}\right]\left[\begin{array}{c}
w(k) \\
\eta_{b}(k)
\end{array}\right] \\
y(k) & =\left[\begin{array}{ll}
H_{b} & C_{b}
\end{array}\right]\left[\begin{array}{l}
X_{b}(k) \\
Z_{b}(k)
\end{array}\right] .
\end{aligned}
$$

As can be seen, both systems in (17) and (20) are driven by zero mean white processes and further there is no measurement noise in the output equations. Consequently, the state vectors $X_{f}(k)$ and $X_{b}(k)$ must be estimated using standard reduced order estimators. A treatment of reduced order estimators can be found in [12].

\section{CONCLUSION}

The problem of noncausal vector modeling of images is addressed in this brief. The noncausal vector image model was arranged into descriptor system which was in turn decomposed into equivalent stable forward and backward subsystems. These subsystems were used to design Kalman filters in which the forward state was estimated with the backward state considered as a colored output noise and similarly the backward state was estimated with the forward state regarded as colored output noise. The estimation of the forward and backward states can then be accomplished using standard reduced order estimators. A numerical example was given to demonstrate the decomposition process into stable forward and backward subsystems. 


\section{REFERENCES}

[1] A. K. Jain, "Advances in mathematical models for image processing," Proc. IEEE, vol. 69, pp. 502-528, May 1981.

[2] B. R. Suresh and B. A. Shenoi, "New results in two-dimensional Kalman filtering with applications to image restoration," IEEE Trans. Circuits Syst., vol. CAS-28, pp. 307-319, Apr. 1981.

[3] J. W. Woods and C. H. Radewan, Kalman Filtering in Two Dimensions, IEEE Trans. Inform. Theory, vol. IT-23, pp. 473-482, July 1977.

[4] M. R. Azimi-Sadjadi, "Strip Kalman filtering for image restoration: New results,", IEEE Trans. Circuits Syst., vol. 37, pp. 284-290, Feb. 1990

[5] D. G. Luenberger, "Dynamic systems in descriptor form," IEEE Trans. Automat. Contr., vol. AC-22, pp. 312-321, June 1977.

[6] G. C. Verghese, B. C. Levy, and T. Kailath, "A generalized state space for singular systems," IEEE Trans. Automat. Contr., vol. AC-26, pp. 811-831, Aug. 1981.

[7] A.-C. Hsueh, "State variable modeling and recursive processing of 1-D and 2-D noncausal systems," Ph.D. dissertation, Univ. Southern California, Aug. 1983.

[8] G. Sharma and R. L. Kashyap, "Two-dimensional spectrum estimation using noncausal autoregressive models," IEEE Trans. Inform. Theory, vol. IT, no. 2, pp. 268-275, Mar. 1986.

[9] G. Lele and Jerry M. Mendel, "Modeling and recursive state estimation for two-dimensional noncausal filters with applications in image restoration," IEEE Trans. Circuits Syst., vol. CAS-34, pp. 1507-1517, Dec. 1987.

[10] J. Biemond, F. G. Van Der Putten, and J. W. Woods, "Identification and restoration of images with symmetric noncausal blurs," IEEE Trans. Circuits Syst., vol. 35, pp. 385-393, Apr. 1988.

[11] M. A. Hasan, "Noncausal image modeling with applications to image restoration," M.S. thesis, Colorado State University, Dec. 1992.

[12] B. D. O. Anderson and J. B. Moore, Optimal Filtering. Englewood Cliffs, NJ: Prentice-Hall, 1979.

\section{Discrete Orthogonal Polynomial Deconvolution for Time-Varying Systems}

Edward B. Moody

\begin{abstract}
Discrete orthogonal polynomial deconvolution (DOPD) has been demonstrated to be a robust method for obtaining the inverse solution for time-invariant systems. In this communication, extension of the method to time-varying linear systems is explored. The operator-based nature of DOPD lends itself to application to linear time-varying systems expressible as an operator matrix. The stability and noise tolerance characteristics of time-invariant DOPD are demonstrated to apply to time-varying systems. $A$ priori estimation of the quality of the inverse solution is possible if the characteristics of noise in the forward solution can be estimated. For time-varying linear systems having a region of basis function support approximately congruent to the support region of the transfer function, and for which there is sufficient a priori knowledge of the system, DOPD provides an efficient and noise tolerant method of inverse solution.
\end{abstract}

\section{INTRODUCTION}

Inverse solution of linear time varying systems is an ill-conditioned problem which requires constrained or approximate solutions [1], [2]. Methods of solution may involve reduction of the time-varying

Manuscript received July 30, 1993; revised June 28, 1994. This paper was recommended by Associate Editor B. Kim.

The author is with the Division of Nuclear Medicine, Department of Diagnostic Radiology, University of Kentucky College of Medicine, Lexington, KY 40536 USA.

IEEE Log Number 9413187. system into time-invariant segments [3]-[5], and tend to be computationally intensive due to matrix inversion or the numerical demands of filter design [3]-[9]. Presented in this communication is the extension of the discrete orthogonal polynomial deconvolution method [10], [11] to linear time-varying systems. The stability and robustness associated with this method in the time-invariant case is demonstrated to extend to time-varying systems.

II. Discrete ORTHOgonal POlynomial

DECONVOLUTION FOR TIME-VARYING LINEAR SYSTEMS

Implementation of DOPD in matrix operator format for timeinvariant systems has been described [10], and will be shown to extend to linear time-varying systems. A discrete linear time-varying system may be expressed in matrix operator format as

$$
\sum_{k, n} B_{k n} O_{n}=I_{k}
$$

Where $I$ is a row vector of length $N$ resulting from the multiplication of the $N \times N$ matrix operator $\mathbf{B}$ with the column vector $O$. The matrix operator $\mathbf{B}$ is constructed using the time-varying system transfer function $h_{k n}$ so that the equation

$$
I_{k}=\sum_{n=1}^{N} h_{k n} O_{n} \quad k=1,2,3, \cdots, N
$$

is satisfied.

The cornerstone of DOPD is the adjoint property of square matrix multiplication operators. In general, the adjoint operator is the complex conjugate transpose of the matrix operator $\mathbf{B}$. For strictly real systems, the adjoint operator, $\mathbf{B}^{+}$is the transpose of $\mathbf{B}$, and has the general property that the inner product of any vector $x$ with the output of the operator $\mathbf{B}$ acting on the vector $y$ is equivalent to the inner product of $y$ with the output of the adjoint operator $\mathbf{B}^{+}$acting on $x$ (3) [12], [13].

$$
\begin{aligned}
\sum_{k} x_{k} \cdot \sum_{k, n} B_{k n} y_{n}=\sum_{k} y_{k} \cdot \sum_{k, n} B_{k n}^{+} x_{n} \\
k=1,2,3, \cdots, N \\
n=1,2,3, \cdots, N .
\end{aligned}
$$

When the system function $h$ is time-invariant and symmetrical, $\mathbf{B}$ is block-circulant and self-adjoint $\left(\mathbf{B}^{+}=\mathbf{B}\right)$. However the adjoint property as stated in (3) is generally applicable to linear systems expressible in square matrix form and has no implicit restrictions regarding causality or temporal characteristics of the transfer function. The time-varying characteristics of the system are incorporated into DOPD when the matrix transfer function $\mathbf{B}$ and the adjoint operator $\mathbf{B}^{+}$are cast. Subsequent DOPD operations proceed in identical fashion to the time-invariant case [10], but are repeated here to provide a complete description for the time-varying case.

DOPD is initiated by application of the adjoint operator $\mathrm{B}^{+}$to a set of $I Z$ orthonormal basis functions $p_{m k}$ of length $N$. Hartley functions, defined by

$$
\begin{gathered}
p_{m k}=\sin \frac{2 \pi(m-1) k}{N}+\cos \frac{2 \pi(m-1) k}{N} \\
p_{(m+1) k}=\sin \left(-\frac{2 \pi(m-1) k}{N}\right)+\cos \left(-\frac{2 \pi(m-1) k}{N}\right) \\
k=1,2,3, \cdots, N \\
m=1,3,5, \cdots, I Z-1
\end{gathered}
$$

